Onkologe $2020 \cdot 26: 205-218$

https://doi.org/10.1007/s00761-019-00707-3

Online publiziert: 31. Januar 2020

(c) Der/die Autor(en) 2020

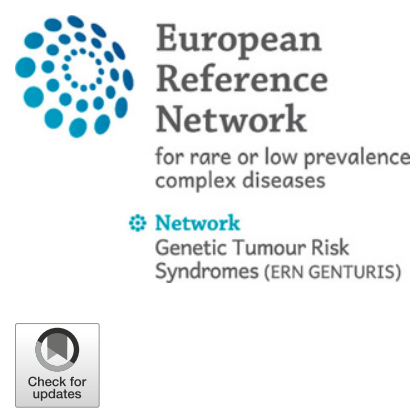

\section{Klinische Relevanz von Tumordispositionssyndromen}

Als erbliche Tumorsyndrome werden Krankheitsbilder mit einem deutlich erhöhten Lebenszeitrisiko für die Entstehung bestimmter, $\mathrm{z}$. T. frühmanifester Tumoren bezeichnet. Die Disposition beruht meist auf einer hochpenetranten Keimbahnmutation in einem einzelnen Gen.

\section{》) Vererbt wird die genetische Disposition für ein erhöhtes Tumorrisiko}

Es handelt sich somit um klassische erblich bzw. genetisch bedingte Krankheitsbilder, die den Mendel-Gesetzen der Vererbung (dominant, rezessiv) folgen. Vererbt wird dabei nicht der Tumor selbst, sondern die genetische Disposition für ein erhöhtes Tumorrisiko; präziser spricht man deshalb von Tumordispositionssyndromen (TDS). In - Abb. 1 ist ein autosomal-dominanter Erbgang am Beispiel des Li-Fraumeni-Syndroms dargestellt. Für Kinder einer betroffenen Person bzw. eines Anlageträgers besteht eine 50\%ige Wahrscheinlichkeit, das mutierte Allel oder das Wildtyp-Allel zu erben.

Bei bis zu $30 \%$ der Patienten mit malignen Erkrankungen beobachtet man eine familiäre Häufung von Tumoren, die

\author{
Claudia Perne ${ }^{1,2} \cdot$ Verena Steinke-Lange $^{3,4,5} \cdot$ Stefan Aretz ${ }^{1,2} \cdot$ Isabel Spier $^{1,2}$ \\ ${ }^{1}$ Institut für Humangenetik, Universitätsklinikum Bonn, Bonn, Deutschland \\ ${ }^{2}$ Nationales Zentrum für erbliche Tumorerkrankungen (NZET), Universitätsklinikum Bonn, Bonn, \\ Deutschland \\ ${ }^{3}$ MGZ - Medizinisch Genetisches Zentrum, München, Deutschland \\ ${ }^{4}$ ZET - Zentrum für Erbliche Tumorerkrankungen, München, Deutschland \\ ${ }^{5}$ Medizinische Klinik und Poliklinik IV, Campus Innenstadt, Klinikum der Universität, München, \\ Deutschland
}

\title{
Seltene Tumoren als Leitsymptom hereditärer Tumorsyndrome
}

an eine erbliche Form denken lässt. Das Vorliegen eines TDS ist deshalb eine häufige Verdachtsdiagnose bei der Erhebung der Eigen- und Familiengeschichte. Etwa $5 \%$ aller (soliden) Krebserkrankungen beruhen auf einer monogen erblichen Veranlagung; je nach Tumortyp und Erkrankungsalter kann der Anteil aber deutlich höher liegen [14]. Damit treten in Deutschland jährlich mindestens 20.000 Malignome im Rahmen eines TDS auf.

Die Erkennung und korrekte Einordnung monogen erblicher Tumorformen ist von hoher klinischer Relevanz, da Patienten, Risikopersonen und asymptomatische Anlageträger eine im Vergleich zu Patienten mit sporadischen Krebserkrankungen spezielle, intensivere und langfristigere medizinische Betreuung benötigen. Einerseits besteht ein hohes Lebenszeitrisiko für Tumoren eines bestimmten und z. T. breiten Spektrums sowie ein hohes Wiederholungsrisiko bei verwandten Familienangehörigen; andererseits ist durch intensivierte Vorsorgeund Früherkennungsuntersuchungen sowie chirurgische Maßnahmen häufig eine effiziente Krebsprävention möglich. Zum Teil bestehen auch spezifische medikamentöse Therapieansätze.

Hereditäre Tumorformen stehen deshalb paradigmatisch für ein äußerst erfolgreiches Konzept der präventiven Onkologie und individualisierten Medizin. Sie begegnen dem Arzt in jeder Alters- gruppe und zeigen eine mitunter ausgeprägte klinische Variabilität, auch innerhalb einer Familie. Für die professionelle Betreuung der Patienten und ihrer Angehörigen ist in besonderem Maße eine multidisziplinäre Zusammenarbeit zwischen Humangenetik, Pathologie und verschiedenen klinischen Disziplinen notwendig. Spezialisierte interdisziplinäre Zentren sollten deshalb in Diagnostik und Koordination der Behandlung und Früherkennung eingebunden sein.

\section{Klinische Hinweise, Differenzialdiagnostik}

Für die Identifizierung häufigerer bzw. charakteristischer TDS wurden spezifische diagnostische Kriterien entwickelt, die beispielsweise bei der Website GeneReviews nachzulesen sind (•Infobox 1 ). Allgemeine klinische Hinweise für ein TDS sind in • Infobox 2 aufgeführt [1, 10].

Bei Verdacht auf Vorliegen eines TDS ist es sinnvoll, den Patienten und seine Familienangehörigen in einer humangenetischen Sprechstunde vorzustellen [7] (-Infobox 1), wo mittels Stammbaumanalyse und Beurteilung klinischer Informationen eine differenzialdiagnostische Einschätzung erfolgt (• Abb. 2). Je nach Verdachtsdiagnose können dann ggf. die genetische Diagnostik eingeleitet und zusammen mit anderen klinischen Fachbereichen individuell angemessene 


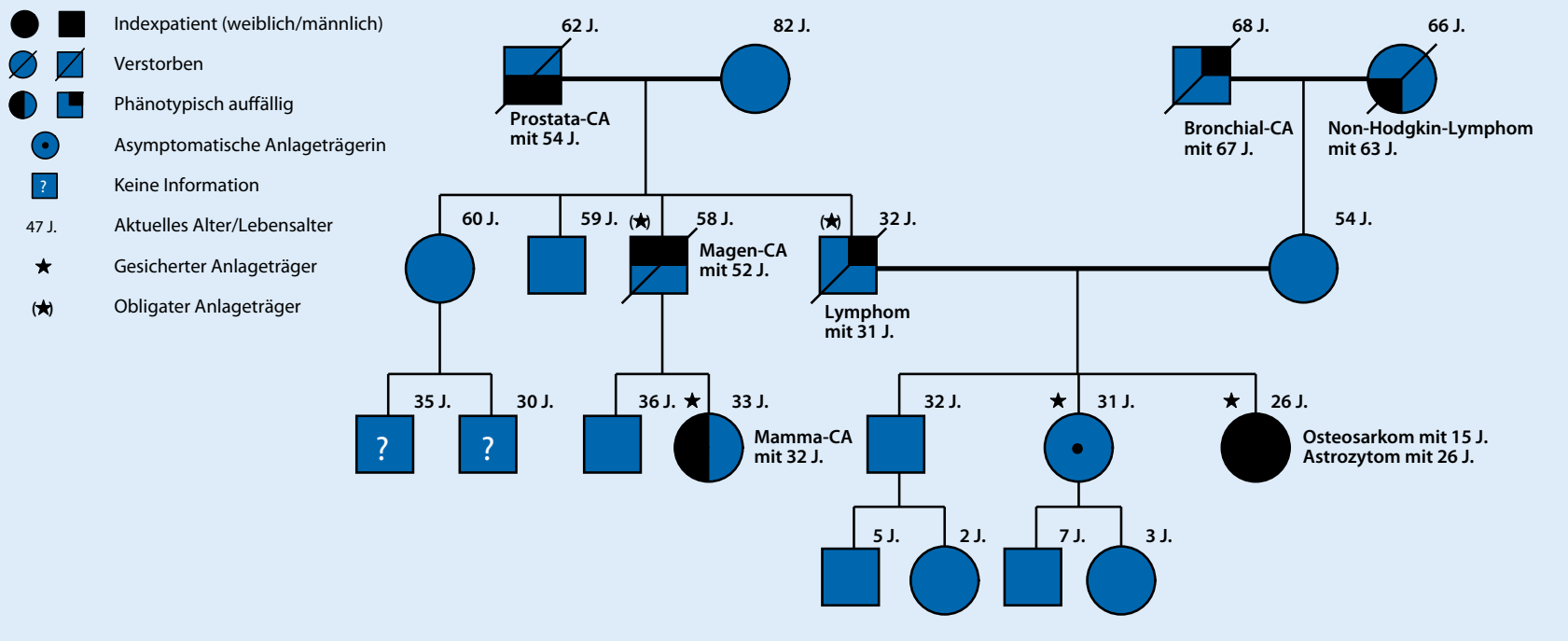

Abb. 1 ॥ Autosomal-dominanter Erbgang am Beispiel des Li-Fraumeni-Syndroms mit Nachweis der heterozygoten Keimbahnmutation c.818G >A; p.(Arg273His) im TP53-Gen. Erläuterung s. Fallbeispiel. CA Karzinom

(„risikoadaptierte“) Empfehlungen für Vorsorge- bzw. Früherkennungsuntersuchungen gegeben werden sowie weitere Risikopersonen in der Familie benannt werden.

Die seit Langem bekannte unzureichende Identifizierung und medizinische Betreuung der von TDS betroffenen Familien liegt insbesondere an der mangelnden Sensitivität etablierter klinischer Kriterien, komplexer diagnostischer Pfade, der unzureichenden Erhebung aussagekräftiger Familienanamnesen im klinischen Alltag, der eingeschränkten Qualität familienanamnestischer Angaben und kleiner Familien sowie der vergleichsweise noch geringen ärztlichen Aufmerksamkeit der Problematik gegenüber.

\section{Seltene Tumoren versus seltene TDS}

Als selten wird in diesem Beitrag eine Tumorerkrankung mit einer jährlichen Inzidenz von weniger als 6 von 100.000 Personen (entsprechend weniger als 5000 Betroffene in Deutschland) bezeichnet (Daten des Robert Koch-Instituts).

Bei vielen seltenen TDS wird das Tumorspektrum wesentlich von häufig vorkommenden Karzinomen bestimmt; so z. B. vom Mammakarzinom beim LiFraumeni-Syndrom (LFS) oder PTENHamartom-Tumor-Syndrom (PHTS).
Andererseits können sehr seltene Tumoren auf häufigere TDS hinweisen: Talgdrüsenneoplasien oder das Dünndarmkarzinom finden sich beispielsweise beim erblichen Darmkrebs („hereditary nonpolyposis colorectal cancer", HNPCC/Lynch-Syndrom); das Rhabdomyosarkom gehört zum Tumorspektrum der Neurofibromatose Typ 1.

\section{》) Seltene spezifische Tumoren treten relativ häufig im Rahmen eines TDS auf}

Insbesondere seltene spezifische Tumoren treten relativ häufig im Rahmen eines TDS auf. Sie stellen deshalb diagnostisch wichtige Leittumoren zur Identifizierung einer hereditären Ätiologie dar und sollten an das mögliche Vorliegen eines TDS denken lassen [1, 10]. So finden sich dem LFS zugrunde liegende TP53Keimbahnmutationen bei etwa $50 \%$ von Kindern mit einem Nebennierenrindenkarzinom oder Plexus-chorioideusKarzinom und bei bis zu $70 \%$ von Kindern mit Rhabdomyosarkomen vom embryonal-anaplastischen Subtyp [6]. Bei etwa $30 \%$ der Patienten mit einem medullären Schilddrüsenkarzinom lässt sich eine RET-Keimbahnmutation nachweisen und damit die Diagnose einer multiplen endokrinen Neoplasie Typ 2 (MEN2) stellen.
Das Auftreten eines seltenen Tumors rechtfertigt deshalb oft bereits die genetische Abklärung mittels Keimbahndiagnostik, auch ohne auffällige Familienanamnese. Eine Auswahl klinisch relevanter, seltener, überwiegend maligner Tumoren mit den wichtigsten, differenzialdiagnostisch zu berücksichtigenden TDS findet sich in - Tab. 1, eine Beschreibung der entsprechenden TDS in - Tab. 2.

\section{Somatische versus Keimbahnmutation}

In jedem Tumor treten während der Tumorgenese im neoplastischen Gewebe entstandene Mutationen auf, die z. T. als sog. Driver-Mutationen die Tumorentwicklung vorantreiben (,jeder Krebs ist genetisch"). Diese somatischen Mutationen sind in der Regel auf den Tumor begrenzt und verschwinden mit der erfolgreichen Behandlung bzw. Entfernung der Neoplasie. Demgegenüber handelt es sich bei den für TDS ursächlichen genetischen Veränderungen um Keimbahnmutationen (konstitutionelle Mutationen), die in der Regel von einem Elternteil vererbt wurden und in allen Körperzellen des Betroffenen/Anlageträgers vorliegen. Keimbahnmutationen können jedoch auch bei dem Betroffenen bzw. in einer Keimzelle der Eltern neu (de novo) 
Hier steht eine Anzeige.

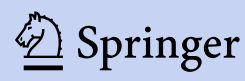


entstehen; häufig ist die Familienanamnese dann unauffällig.

Die bei der genetischen Analyse eines Tumors nachgewiesenen Mutationen erlauben in der Regel keine sichere Unterscheidung zwischen einer somatischen, nur im Tumor selbst auftretenden und einer konstitutionellen Mutation - für den sicheren Nachweis einer Keimbahnmutation im Rahmen der Diagnostik eines TDS ist deshalb die Untersuchung von gesundem Gewebe notwendig, meistens anhand von Leukozyten-DNA einer EDTA-Blutprobe.

\section{Molekulargenetische Diagnostik}

TDS beruhen auf Keimbahnmutationen in einem von derzeit über 100 bekannten Genen [16], (CancerGeneCensus, - Infobox 1). Die molekulargenetische Untersuchung eines oder mehrerer Gene in einer Familie sollte - wann immer möglich - zuerst bei einer sicher erkrankten Person (Indexpatient) gezielt auf der Basis einer möglichst präzisen klinischen Verdachtsdiagnose erfolgen. Inzwischen werden hierzu fast ausschließlich auf Hochdurchsatztechniken (Next-Generation-Sequencing; NGS) basierende Multi-Gen-Analysen („GenPanel“) eingesetzt, bei denen alle bekannten, sicher ursächlichen Gene des Krankheitsbilds sowie die Gene relevanter Differenzialdiagnosen simultan oder als Stufendiagnostik untersucht werden.

Je typischer das klinische Bild ist, desto größer ist die Wahrscheinlichkeit, eine kausale Keimbahnmutation zu identifizieren. Eine erfolglose Mutationssuche stellt allerdings eine klinisch eindeutige Diagnose nicht infrage; so können beispielsweise Mutationen in intronischen oder regulatorischen Bereichen der Gene oder Mutationsmosaike heute oft noch nicht routinemäßig erkannt und interpretiert werden.

Durch den Einsatz breiter MultiGen-Analysen bei Verdacht auf Vorliegen eines häufigen TDS (z.B. des erblichen Brust- und Eierstockkrebses) werden inzwischen auch seltene TDS wie das LFS vermehrt außerhalb klassischer etablierter klinischer Kriterien

Onkologe $2020 \cdot 26: 205-218$ https://doi.org/10.1007/s00761-019-00707-3

(c) Der/die Autor(en) 2020

\section{Perne $\cdot$ V. Steinke-Lange $\cdot S$. Aretz $\cdot$ I. Spier}

\section{Seltene Tumoren als Leitsymptom hereditärer Tumorsyndrome}

\section{Zusammenfassung}

Hintergrund. Monogen erbliche Tumorsyndrome bzw. Tumordispositionssyndrome (TDS) beruhen auf Keimbahn-/konstitutionellen Mutationen in Schlüsselgenen der Karzinogenese. Ein frühes Erkrankungsalter und eine Häufung von Tumoren eines typischen Spektrums in der Eigen- und/oder Familienanamnese sind Hinweise für das Vorliegen einer hereditären Form. Insbesondere seltene spezifische Tumoren treten relativ häufig im Rahmen eines TDS auf. Methode. Der vorliegende Artikel stellt basierend auf einer Literaturrecherche dar, welche TDS bei Vorliegen eines seltenen Tumors als Differentialdiagnose (DD) bedacht werden sollten.

Ergebnisse. Die Identifizierung einer ursächlichen Keimbahnmutation bei einer erkrankten Person der Familie dient der DD, Ermittlung des Wiederholungsrisikos und der prädiktiven Testung asymptomatischer Risikopersonen. Bei TDS mit autosomaldominantem Erbgang lassen sich oft mehrere Hochrisikopersonen in den betroffenen Familien identifizieren.
Schlussfolgerung. Die frühe Erkennung und korrekte Einordnung ist von hoher klinischer Relevanz, da den Erkrankten und Risikopersonen häufig effektive präventive Maßnahmen (FrüherkennungsUntersuchungen, prophylaktische Operationen) angeboten werden können und z. T. spezielle therapeutische Optionen bestehen. TDS stehen paradigmatisch für ein äußerst erfolgreiches Konzept der präventiven Onkologie und personalisierten Medizin. Die Einführung neuer Methoden der Hochdurchsatz-Sequenzierung (NextGeneration-Sequencing) ermöglicht eine effektivere genetische Diagnostik, stellt aber auch eine Herausforderung für die Befundinterpretation und Beratung dar. Für die Betreuung der Familien ist die Anbindung an multidisziplinäre Expertenzentren sinnvoll.

Schlüsselwörter

Monogen erbliche Tumorsyndrome · Tumordispositionssyndrome · Früherkennung · Keimbahnmutation · Gen-Panel

\section{Rare tumors as a leading symptom of hereditary tumor syndromes}

\section{Abstract}

Background. Monogenic hereditary tumor syndromes or tumor disposition syndromes (TDS) are based on germline/constitutional mutations in key genes of carcinogenesis. Early-onset and a clustering of tumors belonging to a typical spectrum in the personal or family history are indicators for a hereditary form. In particular, rare specific tumors occur relatively frequently in the context of TDS.

Methods. Based on a literature search, the current article presents information on which TDS should be considered for differential diagnosis (DD) in the presence of a rare tumor. Results. The identification of a causal germline mutation in the index patient is important for the DD, the evaluation of recurrence risks, and predictive testing of asymptomatic at-risk family members. In TDS with autosomal dominant inheritance, it is often possible to identify several high-risk individuals in the affected families.
Conclusion. Early detection and correct classification are of high clinical relevance, since the patients and persons at risk can often be offered effective preventive procedures (surveillance, prophylactic operations), and in some cases, special therapeutic options exist. TDS are paradigmatic for an extremely successful concept of preventive oncology and personalized medicine. The introduction of new methods of highthroughput sequencing (next generation sequencing) enables a more effective genetic diagnosis, but also poses a challenge for the interpretation of findings and counseling. Referral to multidisciplinary expert centers is useful for care of the families.

Keywords Monogenic hereditary tumor syndromes . Tumor disposition syndromes - Surveillance . Germline mutation · Gene panel 


\section{Infobox 1 Mehr Informationen zum Thema: Internetlinks}

Datenbanken/Informationen zu Tumordispositionssyndromen (TDS)

- Cancer Gene Census: https://cancer.sanger.ac.uk/census

- Checkliste Krebsdispositionssyndrome Kinder: www.krebsgesellschaft.de/zertdokumente. html Kinderonkologische Zentren - Checkliste zur Erfassung des genetischen Krebsrisikos bei pädiatrisch-onkologischen Patienten

- Familial Cancer Database (FaCD): www.familialcancerdatabase.nl/default.aspx

- Früherkennungsempfehlungen für Kinder mit TDS: http://clincancerres.aacrjournals.org/ pediatricseries

- GeneReviews/Übersichten zu erblichen Erkrankungen: www.ncbi.nlm.nih.gov/books/ NBK1116/

- National Comprehensive Cancer Network/US-amerikanische Leitlinien Diagnostik und Früherkennung: www.nccn.org

- OMIM (Online Mendelian Inheritance in Man)/Verzeichnis hereditärer Erkrankungen: www. ncbi.nlm.nih.gov/omim?db=OMIM\&itool=toolbar

- Orphanet/Portal für seltene Krankheiten: www.orpha.net

- Pediatric and Adult Rare Tumor Network: www.cancer.gov/pediatric-adult-rare-tumor

- Rare Cancers Europe: www.rarecancerseurope.org

- Übersicht Krebsdispositionssyndrome: www.krebs-praedisposition.de

Europäische Referenznetzwerke; humangenetische Beratungsstellen

- Europäisches Referenznetzwerk (ERN) für erbliche Tumorsyndrome (GENTURIS): www.genturis. eu

- ERN GENTURIS Bonn: Nationales Zentrum für erbliche Tumorerkrankungen am Universitätsklinikum Bonn: http://www.nzet.de

- ERN GENTURIS München: Zentrum für erbliche Tumorerkrankungen am Medizinisch Genetischen Zentrum München: www.erbliche-tumorerkrankungen.de

- ERN GENTURIS Dresden: https://tu-dresden.de/med/mf/kge/patienten-ambulanz/unsereschwerpunkte/familiaere-tumorerkrankungen

- Europäisches Referenznetzwerk (ERN) für seltene Krebserkrankungen mit soliden Tumoren im Erwachsenenalter (EURACAN): http://euracan.ern-net.eu/de/

- Humangenetische Beratungsstellen: www.gfhev.de/de/beratungsstellen/beratungsstellen. php

Patientenvertretungen und Selbsthilfegruppen

- EURORDIS-Patientenvertretung seltener Erkrankungen in Europa: http://www.eurordis.org/de

- Selbsthilfegruppe erblicher Darmkrebs: www.semi-colon.de

- Selbsthilfegruppe familiäre Krebserkrankungen: https://www.brca-netzwerk.de/

- Selbsthilfegruppe Peutz-Jeghers-Syndrom: www.peutz-jeghers.eu

- Selbsthilfegruppe CS/BRRS/PHTS (Cowden-Syndrom/Bannayan-Ruvalcaba-Riley-Syndrom/ PTEN-Hamartom-Tumor-Syndrom): www.shg-cobald.de

- Selbsthilfegruppe Li-Fraumeni-Syndrom: www.Ifsassociation.org/germany

- Selbsthilfegruppe Polyposis: www.familienhilfe-polyposis.de

gezielt oder als Zusatzbefund identifiziert [18]. Daneben spielt die zunehmend umfassendere prätherapeutische somatische Tumordiagnostik im Rahmen individualisierter Therapiekonzepte und nachfolgende Keimbahndiagnostik als separate Eintrittspforte eine immer größere Rolle bei der Identifizierung betroffener Familien.

Die Steigerung der diagnostischen Sensitivität durch eine umfangreiche Keimbahnanalyse bei allen neu diagnostizierten Tumorpatienten unabhängig von klinischen Verdachtsmomenten eines TDS wird insbesondere im pädiatrischen Bereich langsam umgesetzt, wo bis zu $9 \%$ der Betroffenen Anlageträger eines TDS sind, häufig mit unauffälliger Familienanamnese [31]. Der deutlich höheren Detektionsrate stehen derzeit aber auch problematische Aspekte gegenüber, z. B. der Umgang mit bestimmten Mutationstypen (Missense-Varianten, intronische Varianten), deren funktionelle Relevanz derzeit häufig noch nicht sicher einzuschätzen ist (,variants of unknown significance“, VUS).

\section{Prädiktive genetische Testung}

Der Nachweis einer ursächlichen Keimbahnmutation bei einer klinisch betroffenen Person ist entscheidend für die differenzialdiagnostische Abgrenzung
Infobox 2 Allgemeine klinische Hinweise für ein monogen erbliches Tumorsyndrom

- Ungewöhnlich frühes Erkrankungsalter für den Tumor

- Synchrone/metachrone Tumoren bei einer Person

- Familiäre Häufung von Tumoren

- Typisches Spektrum der Tumoren in Eigen-/Familienanamnese

- Seltene Tumoren (• Tab. 1)

Spezifische molekulare Tumorbefunde

und die Einschätzung des Wiederholungsrisikos (Erbgang); darüber hinaus ermöglicht er die sichere prädiktive (vorhersagende) Testung (noch) asymptomatischer Verwandter (• Abb. 1, 2 und 4). Dies bedeutet, dass die aufgrund des Erbgangs hochgradig gefährdeten Familienangehörigen („Risikopersonen“) frühzeitig bezüglich ihres Anlageträgerstatus untersucht werden können. Wird die familiäre Keimbahnmutation bei der Risikoperson ausgeschlossen, besteht im Vergleich zur Allgemeinbevölkerung kein erhöhtes Erkrankungsrisiko und damit keine Notwendigkeit einer spezifischen Vorsorge und entsprechend eine psychische Entlastung. Anlageträger haben hingegen - abhängig von der Penetranz der Mutation - ein hohes Erkrankungsrisiko. Ursächlich unklare Keimbahnvarianten (VUS) können nicht zur prädiktiven Testung genutzt werden.

\section{》) Minderjährige sollen nur bei zeitnahen Konsequenzen prädiktiv getestet werden}

Beiden TDS hat die prädiktive genetische Untersuchung eine neue Dimension der Krebsprävention eröffnet. Die Kenntnis einer erblichen Veranlagung - und damit das Wissen um ein deutlich erhöhtes Erkrankungsrisiko - kann für den Einzelnen und die Familie aber auch mit erheblichen psychosozialen Belastungen verbunden sein. Die prädiktive Diagnostik ist deshalb an strikte Vorgaben gebunden: Sie orientiert sich an den Richtlinien der Bundesärztekammer und dem Gendiagnostikgesetz (GenDG) und wird bei volljährigen Personen nach humangene- 


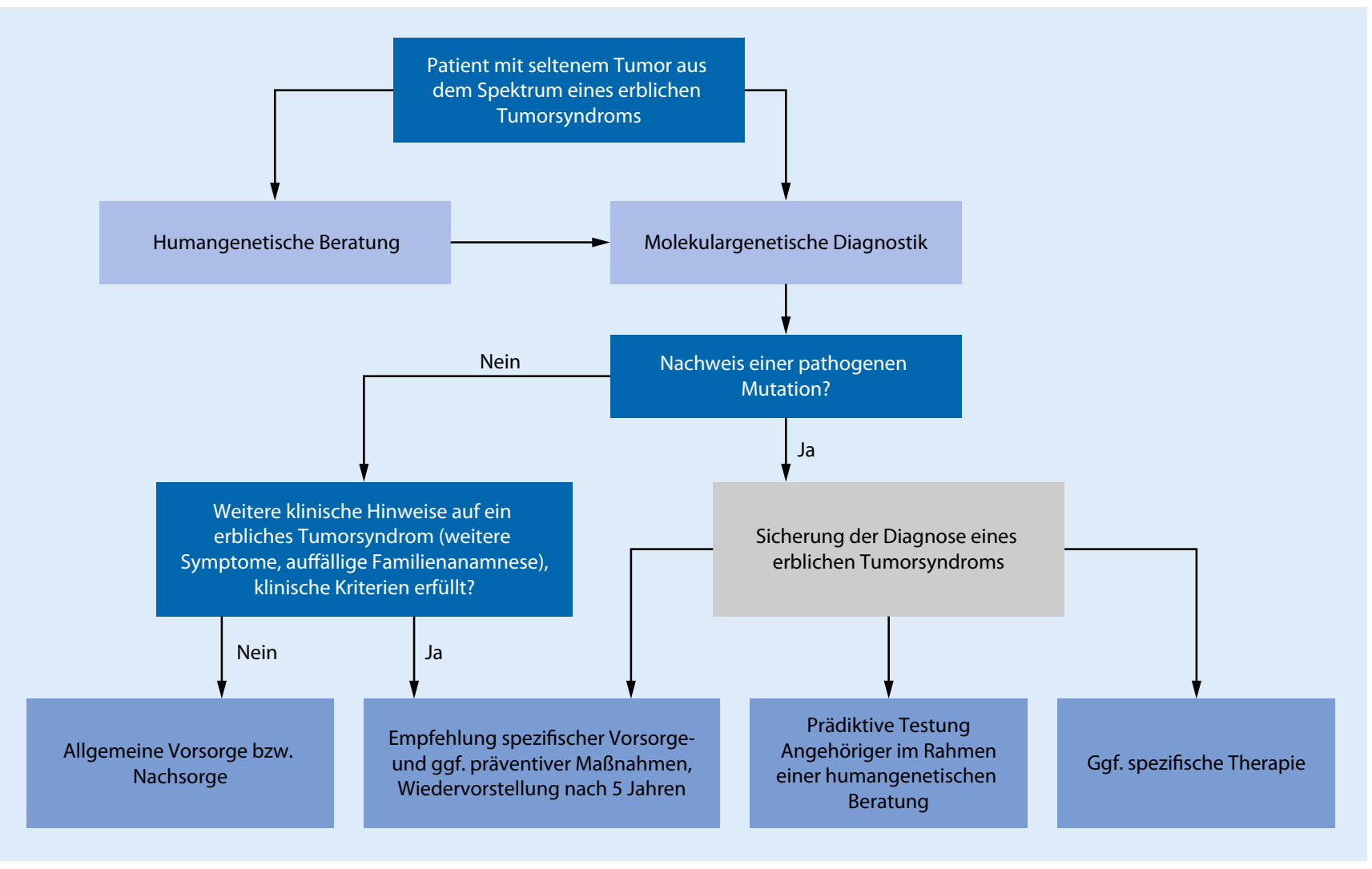

Abb. 2 A Diagnostischer Algorithmus zur Abklärung eines erblichen Tumorsyndroms

tischer Beratung und auffreiwilliger Basis durchgeführt. Ausnahmen sind Risikopersonen für frühmanifeste Erkrankungen. Minderjährige sollen in der Regel aber erst dann prädiktiv getestet werden, wenn sich aus dem Befund zeitnah präventive oder therapeutische Konsequenzen ergeben [9]. Die informationelle Selbstbestimmung des Kindes wird hier über den Wunsch der Eltern nach Kenntnis einer kindlichen Anlageträgerschaft gestellt.

\section{Interdisziplinäre Versorgung/ Prävention/Früherkennung}

Aufgrund des breiten Tumorspektrums und der vielfältigen diagnostischen, präventiven und therapeutischen Maßnahmen lässt sich die adäquate medizinische Versorgung von Familien mit TDS meist nur innerhalb eines interdisziplinären Konzepts realisieren. Für die häufigeren Formen wurden spezifische Vorsorgebzw. Früherkennungsprogramme etabliert, die von gesicherten Mutationsträgern und den Erkrankten selbst sowie
- bei nicht identifizierbarer Mutation in der Familie - von allen erstgradig Verwandten eines klinisch Erkrankten (Risikopersonen) wahrgenommen werden sollten. Für seltene TDS und seltene Manifestationen häufigerer TDS existieren derzeit allerdings meist (noch) keine standardisierten Empfehlungen; es handelt sich in der Regel um Expertenmeinungen bzw. Good Clinical Practice, deren Wirksamkeit aufgrund geringer Fallzahlen schwer zu validieren ist. Aktuelle Empfehlungen finden sich z. B. bei GeneReviews oder dem National Comprehensive Cancer Network (NCCN; - Infobox 1).

\section{Fallbeispiele}

\section{Fallbeispiel Li-Fraumeni-Syndrom}

Bei der Abklärung von Zephalgien wurde bei einer 26-jährigen Patientin (Indexpatientin) in der kranialen MRT ein Astrozytom Grad II diagnostiziert (• Abb. 1). Sie war bereits mit 15 Jahren an einem Osteosarkom des linken Beckens erkrankt, welches kurativ therapiert werden konnte. Aufgrund der auffälligen Anamnese war der Verdacht auf das Vorliegen eines TDS gestellt worden und der Patientin die Vorstellung in der humangenetischen Sprechstunde empfohlen worden.

Die auffällige Familienanamnese mit einem frühmanifesten Lymphom beim Vater und prämenopausalem Brustkrebs bei einer Cousine väterlicherseits ließ in Kombination mit der Eigenanamnese an ein Li-Fraumeni-Syndrom (LFS) denken. Mittels molekulargenetischer Abklärung an Leukozyten-DNA wurde bei der Patientin die pathogene Keimbahnmutation c.818G >A;p.(Arg273His) im TP53-Gen identifiziert und dadurch molekulargenetisch das Vorliegen eines LFS gesichert. Die Diagnose wurde bei der Therapieplanung der Indexpatientin durch Vermeidung einer Strahlentherapie bei erhöhtem Risiko strahleninduzierter Malignome berücksichtigt.

Die Patientin wurde gebeten, ihre Familienangehörigen über die Möglichkeit einer Vorstellung in der humangenetischen Sprechstunde zu informieren, wo 
Tab. 1 Übersicht seltener Tumoren, die im Rahmen von erblichen Tumorsyndromen auftreten können

\section{Seltener Tumor}

Bindegewebstumoren

Mesotheliom

Sarkome ${ }^{\mathrm{a}}$

\section{Dermatologische Tumoren}

Talgdrüsenneoplasien (Adenome, Epitheliome, Karzinome)

Uveamelanom

Endokrinologische Tumoren

Nebennierenrindenkarzinom

Schilddrüsenkarzinom, medullär

Gastrointestinale Tumoren

Cholangiokarzinom

Dünndarmkarzinom

Gastrointestinale Stromatumoren (GIST)

Magenkarzinom, diffus

Gynäkologische Tumoren

Adenoma malignum der Zervix

Keimstrang-Stroma-Tumoren des Ovars

Kleinzelliges Karzinom des Ovars vom hyperkalzämischen Typ (SCCOHT)

Neurologische Tumoren

Astrozytom

Glioblastom

Gliom

Maligner peripherer

Nervenscheidentumor (MPNST)

Medulloblastom

Plexus-chorioideus-Karzinom

Gangliozytom, zerebelläres dysplastisches

\section{Tumordispositionssyndrom (TDS)}

BAP1-Tumorprädipositionssyndrom

Rhabdoidtumor-Prädispositions-Syndrom (RTPS)

Hereditäre Leiomyomatose und Nierenzellkarzinom (HLRCC)

Exostosen/Osteochondrome, multiple hereditäre, Typ 1+2

Li-Fraumeni-Syndrom (LFS)

DICER1-Syndrom

Neurofibromatose Typ 1 (NF1)

Erblicher Darmkrebs (HNPCC/Lynch-Syndrom)

BAP1-Tumorprädipositionssyndrom

Li-Fraumeni-Syndrom (LFS)

Erblicher Darmkrebs (HNPCC/Lynch-Syndrom)

Multiple endokrine Neoplasie, Typ 2A/B (MEN2A/B)

Familiäres medulläres Schilddrüsenkarzinom

Erblicher Darmkrebs (HNPCC/Lynch-Syndrom)

Erblicher Darmkrebs (HNPCC/Lynch-Syndrom)

Peutz-Jeghers-Syndrom (PJS)

Familiäre adenomatöse Polyposis (FAP)

MUTYH-assoziierte Polyposis (MAP)

Hereditäre gastrointestinale Stromatumoren (GIST)

Neurofibromatose Typ 1 (NF1)

Hereditäres diffuses Magenkarzinom (HDGC)

Peutz-Jeghers-Syndrom (PJS)

Peutz-Jeghers-Syndrom (PJS)

DICER1-Syndrom

Rhabdoidtumor-Prädispositions-Syndrom (RTPS)

\section{Li-Fraumeni-Syndrom (LFS)}

Neurofibromatose Typ 2 (NF2)

Erblicher Darmkrebs (HNPCC/Lynch-Syndrom)

Konstitutionelles MMR-Defizienz-Syndrom (CMMRD)

Li-Fraumeni-Syndrom (LFS)

Neurofibromatose Typ 1 (NF1)

Neurofibromatose Typ 2 (NF2)

Neurofibromatose Typ 1 (NF1)

Rhabdoidtumor-Prädispositions-Syndrom (RTPS)

Gorlin-Syndrom

Familiäre adenomatöse Polyposis (FAP)

Li-Fraumeni-Syndrom (LFS)

Li-Fraumeni-Syndrom (LFS)

PTEN-Hamartom-Tumor-Syndrom (PHTS)/Cowden-Syndrom dann ggf. auch eine prädiktive genetische Testung erfolgen kann. Die Mutter der Patientin und ein kinderloser Onkel väterlicherseits wünschten keine genetische Untersuchung. Mit dem Nachweis der TP53-Mutation bei der an Brustkrebs erkrankten Cousine väterlicherseits bestätigte sich dann später die bereits anhand der Familienanamnese naheliegende Vermutung, dass die Indexpatientin die Mutation von ihrem Vater geerbt hat. Die Keimbahnmutation konnte anschlieBend bei einem Bruder der Indexpatientin ausgeschlossen werden; seine Kinder haben somit kein erhöhtes Risiko für ein LFS und bedürfen keiner prädiktiven Testung. Bei der gesunden 31-jährigen Schwester wurde die Mutation hingegen nachgewiesen, sodass ihre 3-jährige Tochter und ihr 7-jähriger Sohn ein 50\%iges Risiko der Anlageträgerschaft aufweisen. Da beim LFS bereits ab der Geburt Früherkennungsuntersuchungen angeboten werden [12, 30], könnte bei den Kindern der Schwester bereits eine prädiktive Testung erfolgen. Die Schwester und ihr Mann sind noch unschlüssig, ob und in welchem Alter die Kinder getestet werden sollen.

Auch wenn noch keine allgemein etablierten Krebsfrüherkennungsuntersuchungen für Patienten mit LFS existieren, wurde den Mutationsträgern der Familie ein engmaschiges, auf wissenschaftlichen Pilotstudien basierendes interdisziplinäres Krebsfrüherkennungsprogramm angeboten, welches u. a. jährliche Ganzkörper-MRT- und SchädelMRT-Untersuchungen vorsieht $[12,30]$. Diese Untersuchungen sollten möglichst an einem interdisziplinären Zentrum für erbliche Tumorerkrankungen im Rahmen von Studien erfolgen.

\section{Fallbeispiel Multiple endokrine Neoplasie Typ 2}

Ein 36 Jahre alter Patient stellt sich auf Empfehlung der Klinik für Endokrinologie mit Verdacht auf das Vorliegen eines TDS in der klinisch-genetischen Ambulanz vor. Bei der Abklärung eines schmerzlosen Knotens im Halsbereich wurde kürzlich ein medulläres Schilddrüsenkarzinom detektiert. Zusätzlich wurde bei erhöhten Kalzium- 


\section{Tab. 1 (Fortsetzung)}

Seltener Tumor

Tumordispositionssyndrom (TDS)

Pädiatrische Tumoren

Hepatoblastom

Familiäre adenomatöse Polyposis (FAP)

Neuroblastom

Hereditäres Neuroblastom

DICER1-Syndrom

Pleuropulmonales Blastom

Wilms-Tumor

DICER1-Syndrom

DICER1-Syndrom

Hereditärer Wilms-Tumor

Urologische Tumoren

Nierenkarzinom, nicht-klarzellig

kalzifizierender (LCCSCT)

Urothelkarzinom (Nierenbecken und Ureter)

Die Aufzählung erhebt keinen Anspruch auf Vollständigkeit, sondern umfasst die nach Ansicht der Autoren wichtigsten Formen. Benigne Tumoren wurden bis auf einzelne Ausnahmen nicht berücksichtigt

z. T. spezifische Sarkome, - Tab. 2

HDGC "hereditary diffuse gastric cancer", hereditäres diffuses Magenkarzinom; HLRCC "hereditary leiomyomatosis and renal cell cancer", hereditäre Leiomyomatose und Nierenzellkarzinom; HNPCC "hereditary nonpolyposis colorectal cancer", erblicher Darmkrebs; MMR "mismatch-repair"; SCCOHT "small-cell carcinoma of the ovary, hypercalcemic type", kleinzelliges Karzinom des Ovars vom hyperkalzämischen Typ

und Parathormonwerten ein Nebenschilddrüsenadenom nachgewiesen. Bis auf einen Hyperparathyreoidismus der Mutter ist die Familienanamnese unauffällig (• Abb. 3 ).

\section{》) Mit einem unauffälligen genetischen Befund ist die Diagnose eines TDS nicht ausgeschlossen}

Die Kombination eines medullären Schilddrüsenkarzinoms und eines $\mathrm{Ne}$ benschilddrüsenadenoms ist hochverdächtig auf das Vorliegen einer multiplen endokrinen Neoplasie Typ 2A (MEN2A). Trotz erfüllter klinisch-diagnostischer Kriterien ließ sich molekulargenetisch keine Keimbahnmutation im RET-Gen identifizieren. Mit dem unauffälligen genetischen Befund ist die Diagnose einer MEN2 aber nicht ausgeschlossen, da mit den Methoden der Routinediagnostik nicht alle denkbaren Mutationen eines Gens nachweisbar sind.
Bei RET-Mutationsträgern richten sich die Empfehlungen bezüglich des Alters prophylaktischer Operationen und des Beginns von biochemischen Früherkennungsuntersuchungen nach der spezifisch vorliegenden Mutation. Ohne Nachweis einer genetischen Ursache bei dem erkrankten Patienten kann den Familienangehörigen keine prädiktive genetische Diagnostik angeboten werden. Erstgradig verwandten Risikopersonen eines Patienten mit genetisch ungeklärter, aber klinisch bestehender MEN2 können aber trotzdem risikoadaptierte Früherkennungsprotokolle, die insbesondere bildgebende und biochemische Untersuchungen umfassen, angeboten werden; eine prophylaktische Thyreoidektomie wird in der Regel allerdings nur genetisch gesicherten Anlageträgern empfohlen.

\section{Fallbeispiel HNPCC/Lynch- Syndrom}

Aufgrund unklarer Oberbauchschmerzen wurde eine 52-jährige Patientin in der internistischen Sprechstunde vorstellig. In der Gastroduodenoskopie zeigte sich ein Karzinom im Duodenum, koloskopisch wurden zwei tubuläre Adenome abgetragen. In der Familienanamnese fiel auf, dass der Vater mit 45 Jahren an Darmkrebs verstorben war. Seine Schwester sei mit 60 Jahren an „Unterleibskrebs“ erkrankt, genauere Informationen hierzu waren nicht erhältlich (• Abb. 4).

Aufgrund des geringen Erkrankungsalters des Vaters waren die revidierten Bethesda-Kriterien erfüllt, sodass klinisch der Verdacht auf ein HNPCC/ Lynch-Syndrom bestand [27]. Zur weiteren Abklärung wurde eine immunhistochemische Untersuchung des Tumorgewebes der Patientin eingeleitet, die einen kombinierten Expressionsverlust der DNA-Mismatch-ReparaturGene MSH2 und MSH6 ergab. Die Patientin stellte sich daraufhin in der humangenetischen Sprechstunde vor, wo anhand einer EDTA-Blutprobe eine $\mathrm{Mu}$ tationssuche in den Genen MSH2 und MSH6 veranlasst wurde. Diese ergab den Nachweis der heterozygoten pathogenen Keimbahnmutation c.942+3A $>\mathrm{T}$ im MSH2-Gen. Das Vorliegen eines HNPCC/Lynch-Syndroms wurde somit bestätigt. Die Patientin wurde daraufhin in ein engmaschiges Vorsorgeprogramm eingeschlossen, welches insbesondere ein- bis zweijährliche Koloskopien und Gastroduodenoskopien beinhaltet [11]. Wegen des erhöhten Endometriumkarzinomrisikos wurde mit ihr die Möglichkeit einer prophylaktischen Hysterektomie besprochen. Im Fall eines Rezidivs der Tumorerkrankung ist eine Behandlung mit PD1-Blockern zu erwägen, da Lynch-Syndrom-assoziierte Tumoren, die hoch immunogen sind, gut auf diese Behandlung ansprechen.

Bei der 30-jährigen Tochter der $\mathrm{Pa}$ tientin wurde die MSH2-Mutation mittels prädiktiver Testung ebenfalls nachgewiesen. Sie wurde in das engmaschige Vorsorgeprogramm eingeschlossen und entschied sich zudem für eine Hormonspirale, um ihr Risiko für ein Endometriumkarzinom zu senken. Ihr 5-jähriger Sohn wird sich im Erwachsenenalter in der humangenetischen Sprechstunde vorstellen. Die Indexpatientin informiert auch ihre Angehörigen väterlicherseits 
Hier steht eine Anzeige.

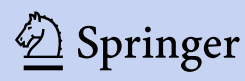




\section{Leitthema}

离|

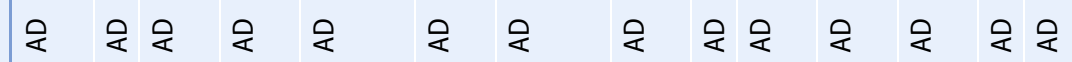

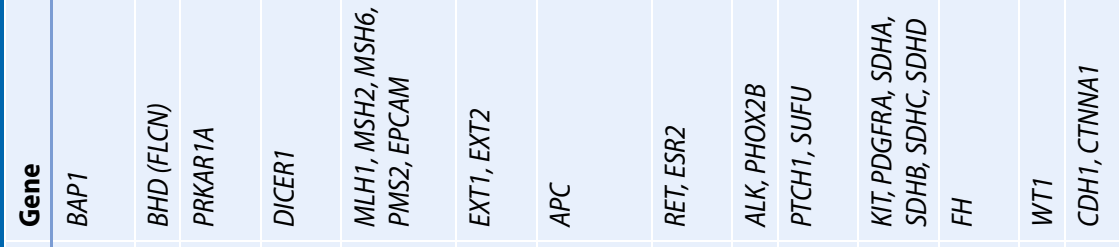
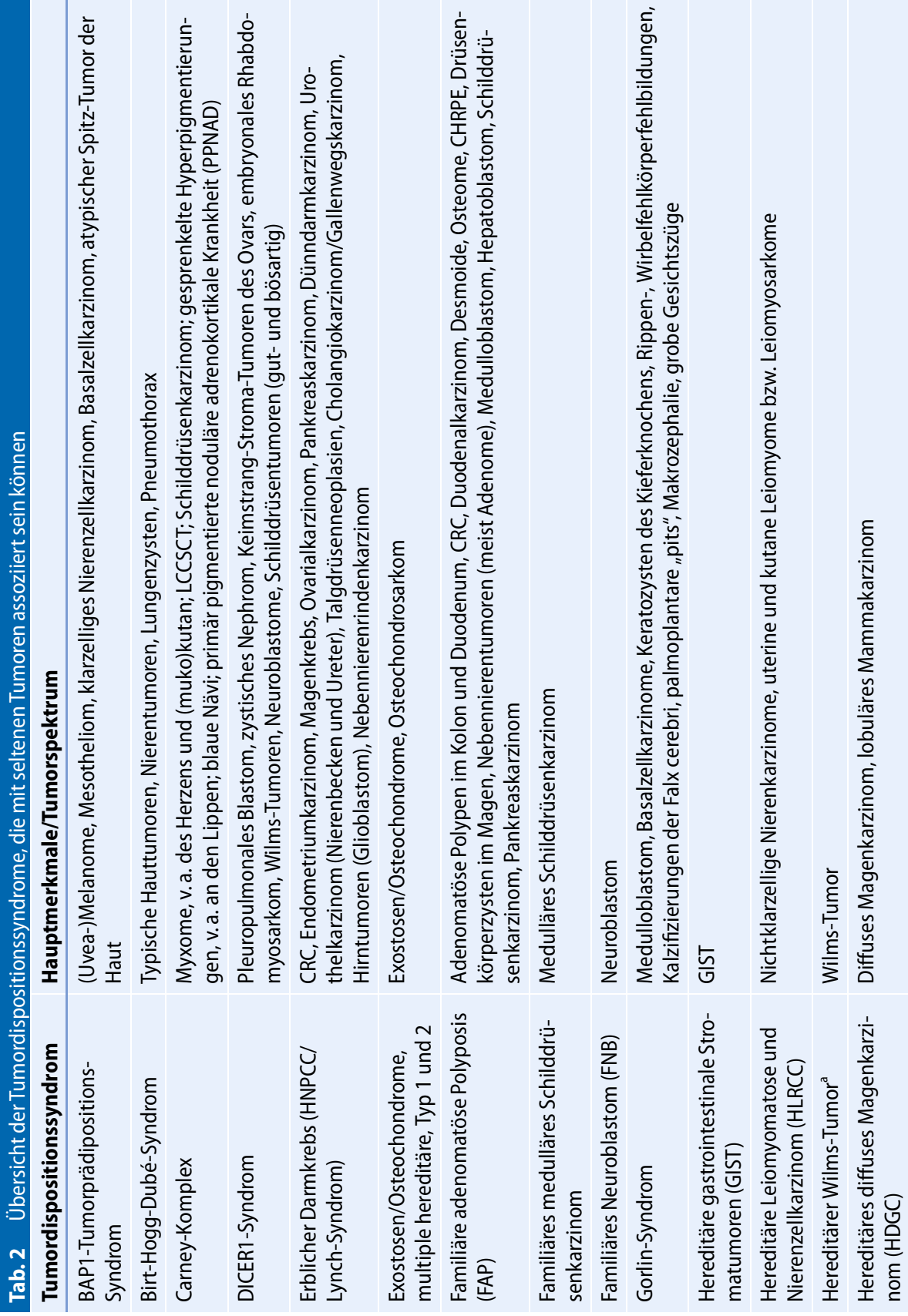


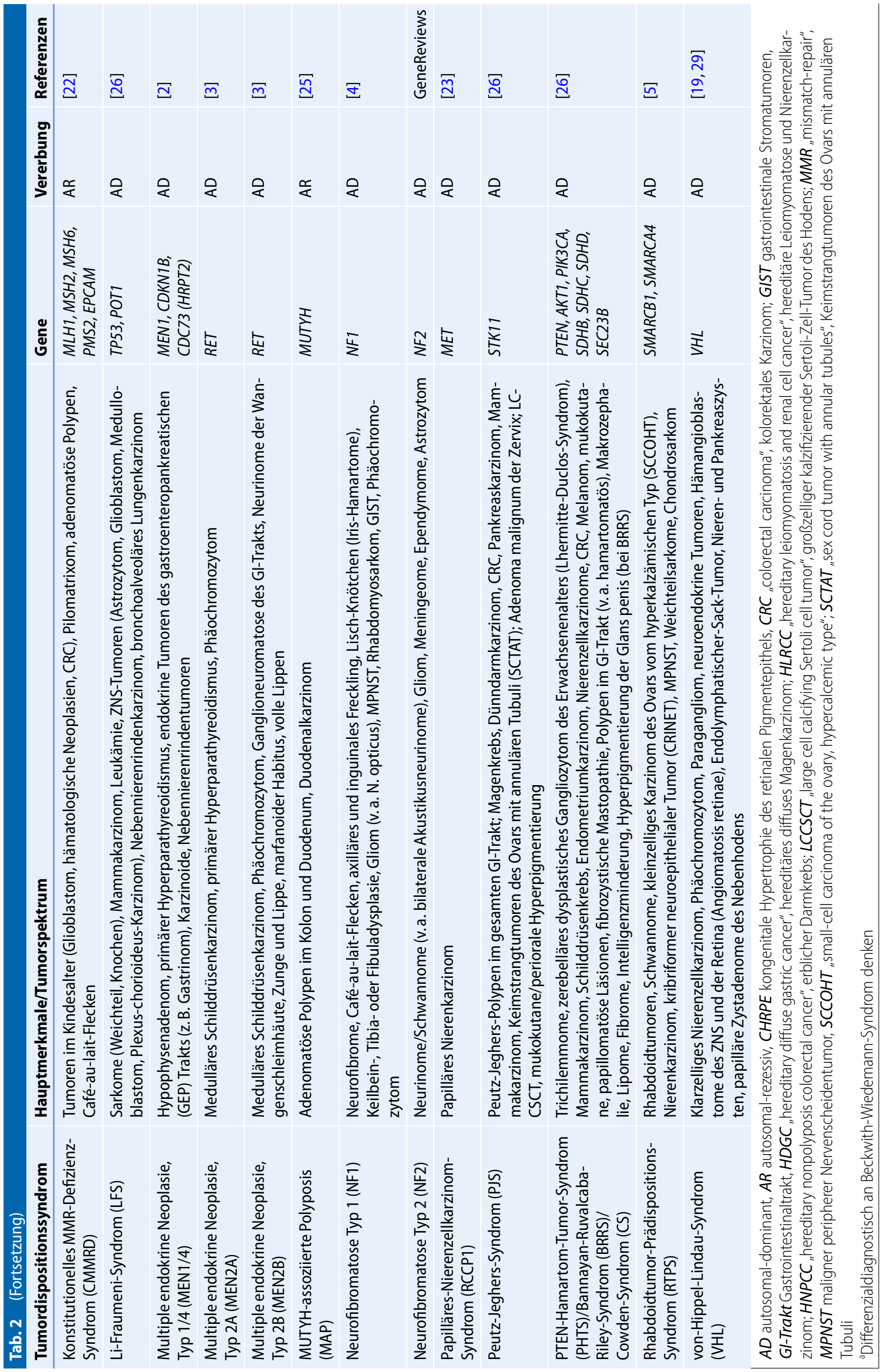




\section{Leitthema}

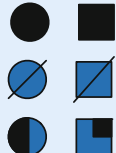

$47 \mathrm{~J}$

Indexpatient (weiblich/männlich)

Verstorben

Phänotypisch auffällig

Aktuelles Alter/Lebensalter

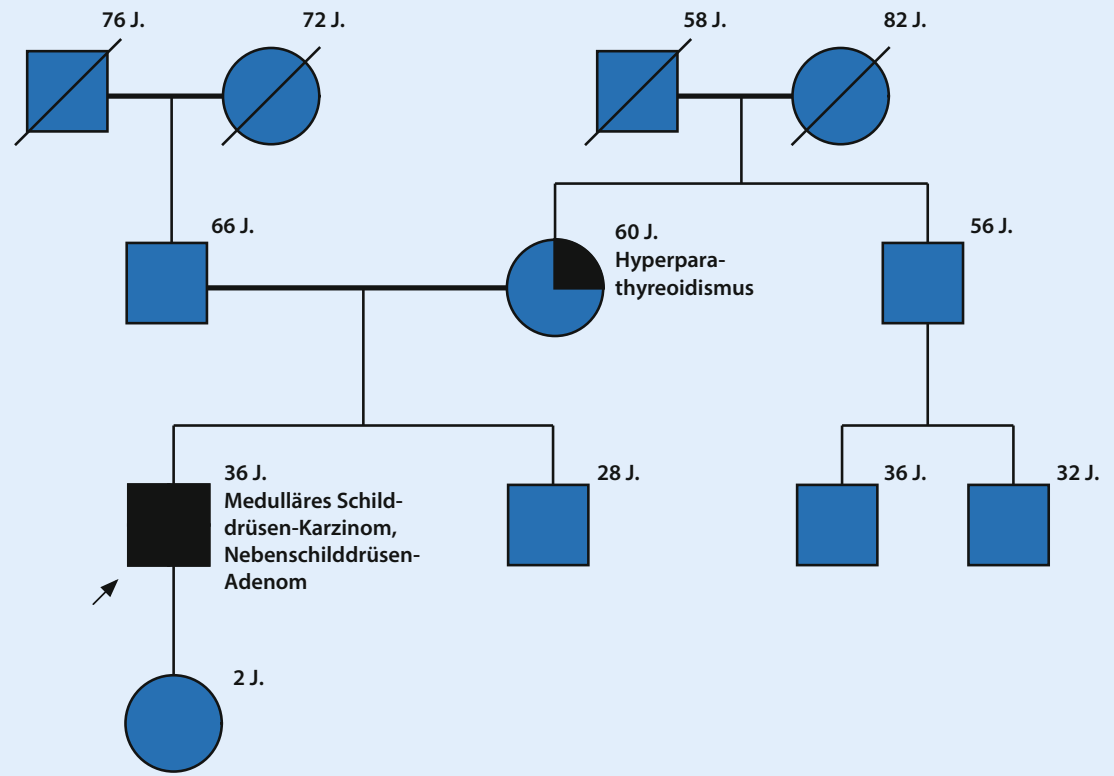

Abb. 3 ॥ Stammbaum zur Fallgeschichte in Bezug auf multiple endokrine Neoplasie Typ 2 (MEN2)

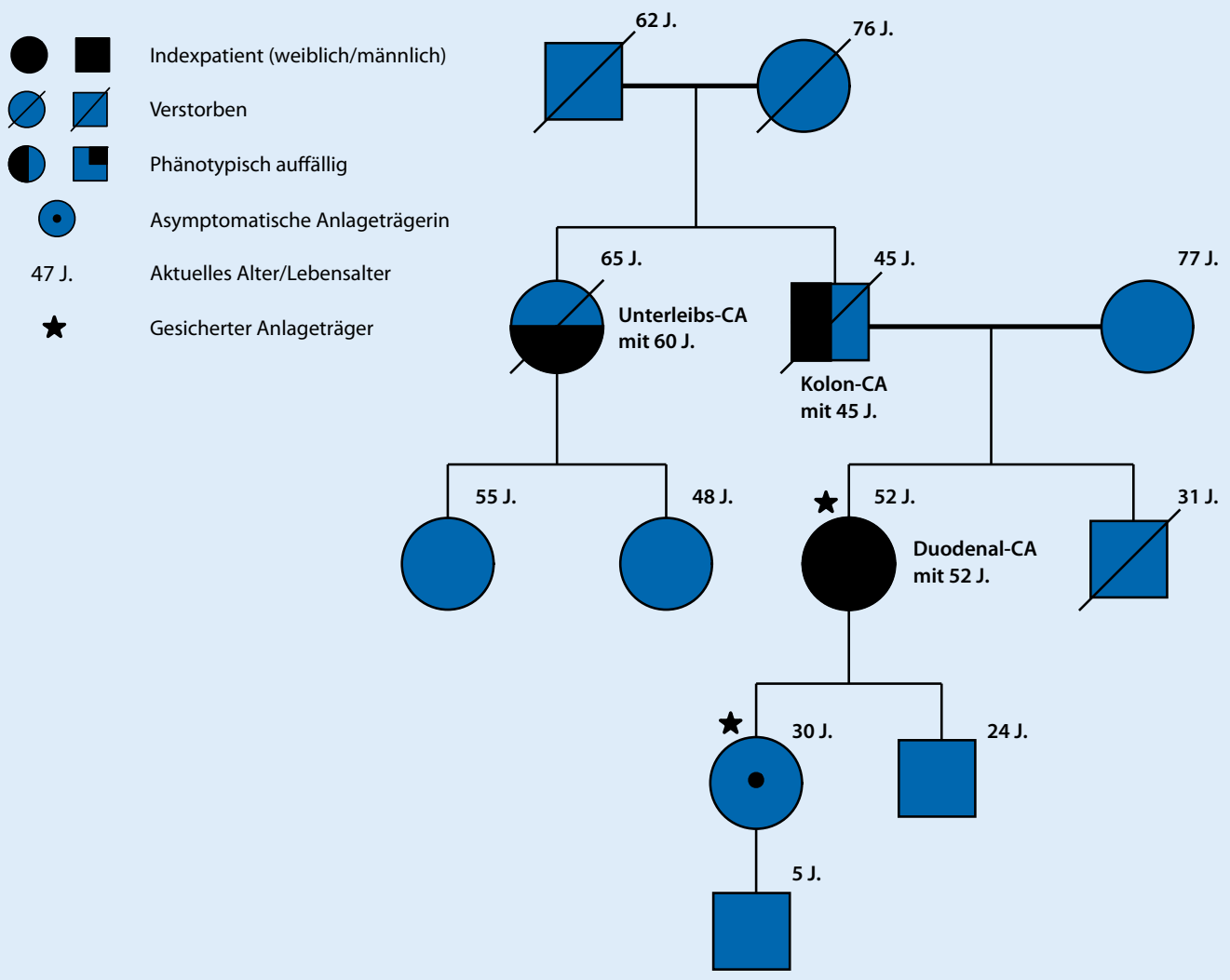

Abb. $4<$ Stammbaum zur Fallgeschichte in Bezug auf erblichen Darmkrebs (,hereditary nonpolyposis colorectal cancer", HNPCC/ Lynch-Syndrom). CA Karzinom 
über die Diagnose einer erblichen Tumorerkrankung in der Familie und die Möglichkeit einer prädiktiven Testung.

\section{Expertenzentren, Vernetzung, Referenznetzwerke}

Nach derzeitigen Schätzungen werden etwa $70-80 \%$ der von TDS betroffenen Patienten nicht erkannt. Eine Chance zur verbesserten Versorgung und Erforschung von TDS ist der Aufbau und die stärkere Vernetzung von Expertenzentren, wie sie auf nationaler Ebene bereits durch Zentren für erbliche Tumorsyndrome bzw. Zentren für seltene Erkrankungen erfolgt. Auf europäischer Ebene wurde als weitere Maßnahme zur Verbesserung der Situation 2017 von der EU-Kommission das europäische Referenznetzwerk für erbliche Tumorsyndrome (ERN GENTURIS - GENetic TUmor RIsk Syndromes) als eines von insgesamt 24 ERN für seltene Erkrankungen etabliert (•Infobox 1$)$.

Das ERN GENTURIS befasst sich mit TDS und vereint die Expertise der beteiligten klinischen Zentren aus derzeit 12 europäischen Ländern. Es soll durch enge Vernetzung der Experten zum einen standardisierte Diagnostik-, Behandlungs- und Präventionskonzepte inklusive interdisziplinärer Fallbesprechungen schaffen sowie Informationsund Lehrmaterialien für Patienten und ihre behandelnden Ärzte bereitstellen. Um die Maßnahmen optimal auf die Bedürfnisse der Patienten abzustimmen, sind Patientenvertreter in alle Arbeitsbereiche eingebunden. Demnächst sollen erste europäischen Leitlinien für zwei seltene TDS, das PHTS und das LFS (TP53-assoziiertes TDS), veröffentlicht und langfristig in die nationalen Leitlinien implementiert werden.

\section{》) Expertenzentren und Selbsthilfegruppen dienen der verbesserten Versorgung von Patienten mit TDS}

Aus Deutschland sind derzeit drei klinische Zentren am ERN GENTURIS beteiligt (-Infobox 1). Diese Zentren fungieren als primärer Ansprechpartner für GENTURIS in Deutschland, sodass sich Ärzte, die hierzulande Patienten mit TDS betreuen, bezüglich Informationen zur Patientenversorgung an diese Zentren wenden können. Für einige seltene TDS existieren bereits überregionale Selbsthilfegruppen, die den Patienten Unterstützung bieten können (- Infobox 1).

\section{Fazit für die Praxis}

- Die Einordnung und korrekte Differenzialdiagnose eines TDS ist entscheidend für die Erfassung der Risikopersonen, Empfehlung angemessener intensivierter Früherkennungsuntersuchungen, präventiver Maßnahmen und Anbindung der Familien an spezialisierte Zentren.

- Die möglichst präzise Verdachtsdiagnose auf der Basis einer angemessenen klinisch-histologischen Untersuchung und sorgfältigen Familienanamnese ist häufig die Voraussetzung einer gezielten rationalen Mutationssuche beim Indexpatienten.

- Das Auftreten eines seltenen Tumors rechtfertigt allerdings oft bereits die genetische Abklärung mittels Keimbahndiagnostik - unabhängig von der Familienanamnese.

- Die prädiktive genetische Diagnostik asymptomatischer Risikopersonen ermöglicht die Beschränkung präventiver Maßnahmen auf die Anlageträger einer Familie und erfolgt im Rahmen einer humangenetischen Beratung.

\section{Korrespondenzadresse}

Dr. med. Isabel Spier
Institut für Humangenetik,
Universitätsklinikum Bonn
Venusberg-Campus 1, Geb.
13,53127 Bonn, Deutschland
isabel.spier@uni-bonn.de

\section{Einhaltung ethischer Richtlinien}

Interessenkonflikt. C. Perne, V. Steinke-Lange, S. Aretz und I. Spier geben an, dass kein Interessen- konflikt besteht. Die Autoren sind am ERN-GENTURIS beteiligt.

Für diesen Beitrag wurden von den Autoren keine Studien an Menschen oder Tieren durchgeführt. Für die aufgeführten Studien gelten die jeweils dort angegebenen ethischen Richtlinien.

Open Access. Dieser Artikel wird unter der Creative Commons Namensnennung 4.0 International Lizenz veröffentlicht, welche die Nutzung, Vervielfältigung, Bearbeitung, Verbreitung und Wiedergabe in jeglichem Medium und Format erlaubt, sofern Sie den/die ursprünglichen Autor(en) und die Quelle ordnungsgemäß nennen, einen Link zur Creative Commons Lizenz beifügen und angeben, ob Änderungen vorgenommen wurden.

Die in diesem Artikel enthaltenen Bilder und sonstiges Drittmaterial unterliegen ebenfalls der genannten Creative Commons Lizenz, sofern sich aus der Abbildungslegende nichts anderes ergibt. Sofern das betreffende Material nicht unter der genannten Creative Commons Lizenz steht und die betreffende Handlung nicht nach gesetzlichen Vorschriften erlaubt ist, ist für die oben aufgeführten Weiterverwendungen des Materials die Einwilligung des jeweiligen Rechteinhabers einzuholen.

Weitere Details zur Lizenz entnehmen Sie bitte der Lizenzinformation auf http://creativecommons.org/ licenses/by/4.0/deed.de.

\section{Literatur}

1. Bashford MT, Kohlman W, Everett J et al (2019) Addendum: a practice guideline from the American college of medical genetics and genomics and the national society of genetic counselors: referral indications for cancer predisposition assessment. Genet Med 21(12):2844. https://doi.org/10.1038/ s41436-019-0586-y

2. Baumgartner-Parzer S (2018) Multiple Endokrine Neoplasie Typ 1 (MEN1). J Klin Endokrinol Stoffw 11:134-135

3. Baumgartner-Parzer S (2018) Multiple Endokrine Neoplasie Typ 2 (MEN2). J Klin Endokrinol Stoffw 11:23-26

4. Baumgartner-Parzer S (2019) Neurofibromatose Typ 1 (NF1-Gen). J Klin Endokrinol Stoffw 12:35-37

5. Bens $S$, Kehrer-Sawatzki $H$, Hasselblatt $M$ et al (2017) SWI/SNF-Komplex-assoziierte Tumordispositions-Syndrome. medgen 29:296-305

6. Bougeard G, Renaux-Petel M, Flaman JM et al (2015) Revisiting Li-Fraumeni syndrome from TP53 mutation carriers. JClin Oncol 33:2345-2352

7. Deutsche Gesellschaft Für Humangenetik (GfH), (BVDH) BDHeV (2011) S2-Leitlinie Humangenetische Diagnostik und genetische Beratung. medgen 23:281-323

8. Figueiredo J, Melo S, Carneiro Pet al (2019) Clinical spectrum and pleiotropic nature of $\mathrm{CDH} 1$ germline mutations. J Med Genet 56:199-208

9. Gendiagnostik-Kommission (2011) Richtlinie der Gendiagnostik-Kommission (GEKO) zu genetischen Untersuchungen bei nicht-einwilligungsfähigen Personen nach § 14 in Verbindung mit $\S 23$ Abs. 2 Nr. 1c GenDG. Bundesgesundheitsbl 54:1257-1261

10. Hartmann A (2017) Pathologie familiärer Tumorsyndrome. Pathologe 38:143-148

11. Hüneburg R, Aretz S, Büttner R et al (2019) Empfehlungen zur Früherkennung, Risikoreduktion, Überwachung und Therapie bei Patienten mit Lynch-Syndrom. Z Gastroenterol 57:1-12 


\section{Leitthema}

12. Kratz CP, Achatz MI, Brugieres L et al (2017) Cancer screening recommendations for individuals with Li-Fraumeni syndrome. Clin Cancer Res 23:e38-e45

13. Langner C (2017) Hereditäres Magen- und Pankreaskarzinom. Pathologe 38:164-169

14. Lu C, Xie M, Wendl MC et al (2015) Patterns and functional implications of rare germline variants across 12 cancer types. Nat Commun 6:10086

15. Patel VM, Handler MZ, Schwartz RA et al (2017) Hereditary leiomyomatosis and renal cell cancer syndrome: an update and review. J Am Acad Dermatol 77:149-158

16. Rahman N (2014) Realizing the promise of cancer predisposition genes. Nature 505:302-308

17. Rai K, Pilarski R, Cebulla CM et al (2016) Comprehensive review of BAP1 tumor predisposition syndrome with report of two new cases. Clin Genet 89:285-294

18. Rana HQ, Gelman R, Laduca H et al (2018) Differences in TP53 mutation carrier phenotypes emerge from panel-based testing. J Natl Cancer Inst 110:863-870
19. Rednam SP, ErezA, Druker Hetal (2017) Von HippelLindau and hereditary pheochromocytoma/ paraganglioma syndromes: clinical features, genetics, and surveillance recommendations in childhood. Clin Cancer Res 23:e68-e75

20. Ricci R (2016) Syndromic gastrointestinal stromal tumors. Hered Cancer Clin Pract 14:15

21. Ripperger T, Schlegelberger B (2018) Genetic predisposition to childhood cancer. Pathologe 39:306-310

22. Ripperger T, Wimmer K, Kratz C (2017) Seltene Tumordispositionssyndrome mit Manifestation im Kindesalter. medgen 29:283-295

23. Schmidt LS, Linehan WM (2016) Genetic predisposition to kidney cancer. Semin Oncol 43:566-574

24. Schultz KAP, Williams GM, Kamihara J et al (2018) DICER1 and associated conditions: identification of atrisk individuals and recommended surveillance strategies. Clin Cancer Res 24:2251-2261

25. Spier I, Hüneburg R, Vilz T et al (2019) Gastrointestinale Polyposis-Syndrome. J Oncol 9:29-42

26. Steinke-Lange V, Becker K, Behnecke A et al (2017) Syndrome mit breitem Tumorspektrum. medgen 29:276-282
27. Steinke V, EngelC, Büttner Ret al (2013) Hereditary nonpolyposis colorectal cancer (HNPCC)/Lynch syndrome. Dtsch Arztebl Int 110(3):32-38

28. Steinlein OK, Ertl-Wagner B, Ruzicka T et al (2018) Birt-Hogg-Dube syndrome: an underdiagnosed genetic tumor syndrome. J Dtsch Dermatol Ges 16:278-283

29. Varshney N, Kebede AA, Owusu-Dapaah H et al (2017) A review of von Hippel-Lindau syndrome. JKidney Cancer VHL 4:20-29

30. Villani A, Shore A, Wasserman JD et al (2016) Biochemical and imaging surveillance in germline TP53 mutation carriers with Li-Fraumeni syndrome: 11 year follow-up of a prospective observational study. Lancet Oncol 17:1295-1305

31. Zhang J, Nichols KE, Downing JR (2016) Germline mutations in predisposition genes in pediatric cancer. NEngl J Med 374:1391

\section{Praxis-Finanzrechner für Ärzte Neues Tool auf SpringerMedizin.de}

Wie rentabel läuft meine Praxis? Wie stehe ich im Vergleich zu meinen Kollegen im Bundesland? Welche Anschaffungen kann ich mir leisten? Antworten auf diese betriebswirtschaftlichen Fragen liefert künftig ein Tool auf SpringerMedizin.de, indem es aktuelle Versorgungs- und Abrechnungsdaten sowie Analysedaten deutscher Gesundheitsunternehmen zu Grunde legt.

Mit den kostenfreien Praxis-Finanzrechnern können sich vor allem in der Praxis tätigen Ärzte, die sich oft auch unternehmerischen Herausforderungen stellen müssen, interaktiv mit den Themen Controlling und Benchmarking auseinandersetzen:

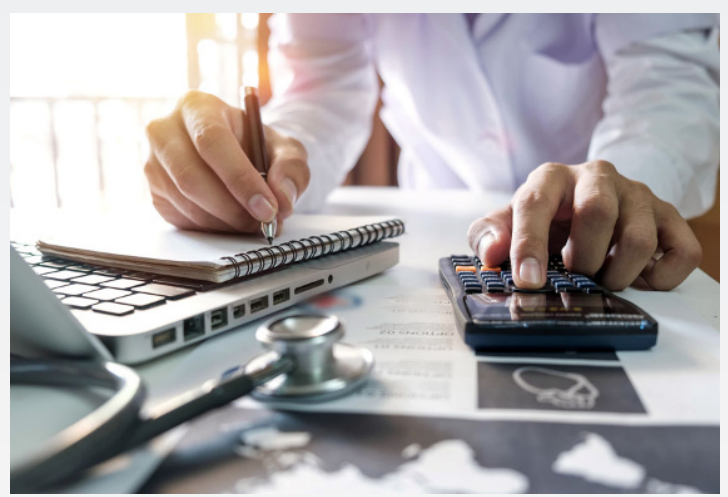

(c) mrmohock/stock.adobe.com
Mit dem Controlling-Assistenten behalten Sie die wichtigsten wirtschaftlichen Praxiskennzahlen immer im Auge. $>$ Mit dem Tool Benchmark können Sie Ihre Praxis mit anderen Praxen vergleichen.

$>$ Ihren Praxisstandort - ebenfalls ein wichtiger Faktor für den Erfolg - analysieren Sie mit Standortcheck aus verschiedenen Perspektiven

Mit dem Investitionsassistenten können sie sehen, ob und wann sich eine Geräteinvestition amortisiert und Sie bekommen auch Anhaltspunkte, welche Einnahmen Sie zu erwarten haben.

Die Praxis-Tools sind ein externes Angebot von Rebmann Research, Partner von SpringerMedizin.de. Sie finden die Praxis-Finanzrechner auf SpringerMedizin.de unter dem Navigationspunkt "Mehr”.

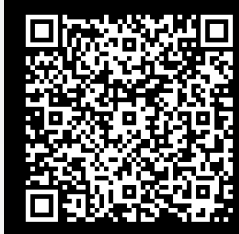

